\title{
Time Localised Band Filtering Using Modified S-Transform
}

\author{
Nithin V George*, Sitanshu Sekhar Sahu*, L. Mansinha ${ }^{\dagger}$, K. F. Tiampo ${ }^{\dagger}$, G. Panda* \\ ${ }^{*}$ Department of Electronics and Communication Engineering, National Institute of Technology, Rourkela, India 769008. \\ ${ }^{\dagger}$ Department of Earth Sciences, University of Western Ontario, London, Canada N6A $5 B 7$. \\ Email: nithinvgeorge@gmail.com, sitanshusekhar@gmail.com, mansinha@uwo.ca , \\ ktiampo@seis.es.uwo.ca, ganapati.panda@gmail.com
}

\begin{abstract}
A noisy time series, with both signal and noise varying in frequency and in time, presents special challenges for improving the signal to noise ratio. A modified S-transform time-frequency representation is used to filter a synthetic time series in a two step filtering process. The filter method appears robust within a wide range of background noise levels.
\end{abstract}

\section{Introduction}

Time series in which both the desired signal and the undesired noise vary with both time and frequency are common to many disciplines. In addition, many time series have added background noise. Fig. 1a shows a synthetic time series $h(t)$, which consists of two chirp signals: one is a time localized chirp and the other one is present across the full length of the time series. Fig. 1b shows the same time series with added uniform gaussian random noise to the level of $-6 \mathrm{~dB}$. Two filter procedures are used to reduce the background noise and the localized chirp signal.

The Fourier amplitude spectrum of $h(t), H(\omega)$ is shown in Fig. 2. Since the energy of the time series is spread across the entire spectra, it is not possible to define a passband and stopband for filtering in the frequency domain.

A more promising solution to the localized filtering problem is the time frequency filter [1][2], based on a joint timefrequency representation (TFR) of the time series, the Stransform.

We introduce a novel filtering approach, which first removes the background noise from the TF plane before applying the TF filter. The $\mathrm{S}$ transform is modified by introducing a different scaling rule for the Gaussian window and is used as the time frequency representation for the filtering. Localized time frequency filtering is simplified by extending image processing tools to time frequency filtering.

\section{The S Transform}

The $\mathrm{S}$ transform is a time frequency analysis technique proposed by Stockwell, Mansinha and Lowe [3], using a Gaussian window whose width scales inversely, and height

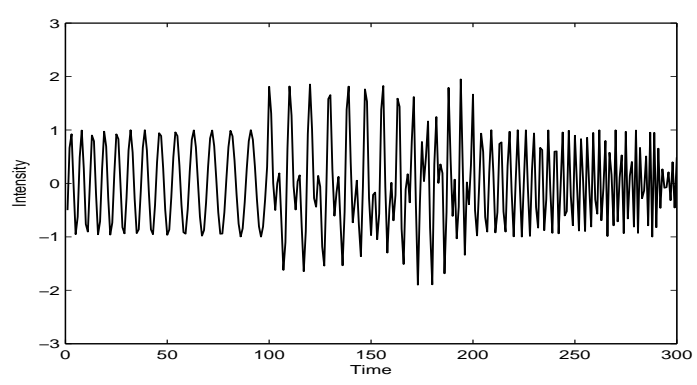

(a) A synthetic time series $h(t)$ is generated by $t=-1: 0.01: 1.99$, $h=\operatorname{chirp}(t, 10,1,20$, 'quadratic' $), t 1=0: 0.01: 1.0, h 1=$ $\operatorname{chirp}\left(t 1,20,1,35\right.$, 'quadratic' $\left.^{\prime}\right), h(100: 200)=h(100: 200)+$ $h 1$

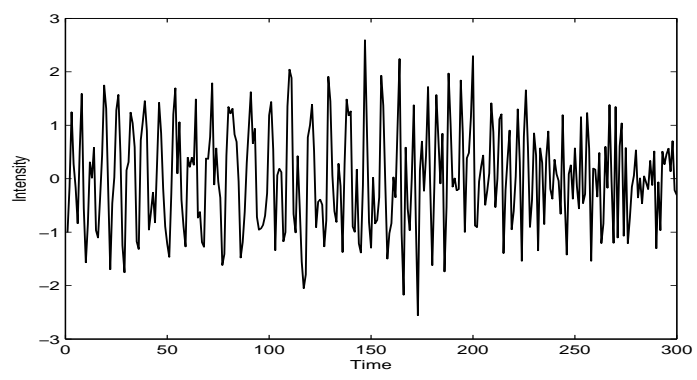

(b) The signal $h(t)$ with an additive noise $(S N R=-6 d B)$

Figure 1.

scales directly with the frequency. It provides frequency dependent resolution while maintaining a direct relationship with the Fourier spectrum. It has been applied in many diverse fields, such as mechanical engineering[4], electrical engineering[5], biomedical instrumentation[6] and geophysics[7]. The $\mathrm{S}$ transform $S(\tau, f)$ of a signal $x(t)$ is defined as

$$
S(\tau, f)=\int_{-\infty}^{\infty} x(t) w(\tau-t) e^{-j 2 \pi f t} d t
$$

where $t$ represents the time, $f$ the frequency, $w$ the scalable gaussian window and the quantity $\tau$ is a parameter which controls the position of the Gaussian window on the $t$-axis. The gaussian window in the time domain is determined by 


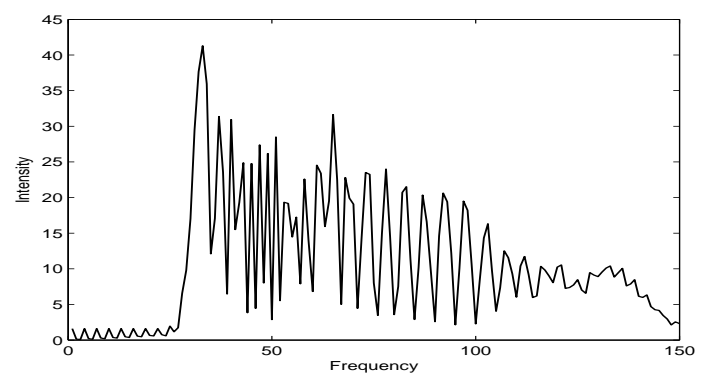

(a) Amplitude spectra of $h(t)$

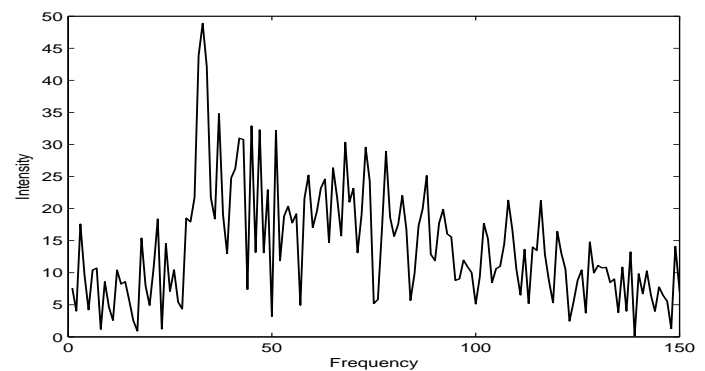

(b) Amplitude spectra of $h(t)$ with an additive noise $(S N R=$ $-6 d B)$

Figure 2.

$$
w(t, \sigma)=\frac{1}{\sigma \sqrt{2} \pi} e^{-\frac{t^{2}}{2 \sigma^{2}}}
$$

where

$$
\sigma(f)=\frac{1}{|f|}
$$

is the scaling scheme used. Combining (2) and (3) gives

$$
S(\tau, f)=\int_{-\infty}^{\infty} x(t)\left\{\frac{|f|}{\sqrt{2} \pi} e^{-\frac{(\tau-t)^{2} f^{2}}{2}} e^{-j 2 \pi f t}\right\} d t
$$

which is the complete definition of original $\mathrm{S}$ transform. The $\mathrm{S}$ transform window satisfies the normalization condition

$$
\int_{-\infty}^{\infty} w(t, f) d t=1
$$

and hence

$$
\int_{-\infty}^{\infty} S(\tau, f) d \tau=X(f)
$$

where $X(f)$ is the Fourier transform of $x(t)$. Averaging $S(\tau, f)$ over all values of $t$ yields $X(f)$, providing a direct link between the S-transform and Fourier transform. The inverse $\mathrm{S}$ transform is given by

$$
x(t)=\int_{-\infty}^{\infty}\left\{\int_{-\infty}^{\infty} S(\tau, f) d \tau\right\} e^{j 2 \pi f t} d f
$$

From equation (4) it can be seen that the time localizing Gaussian translates, while the oscillatory exponential kernel

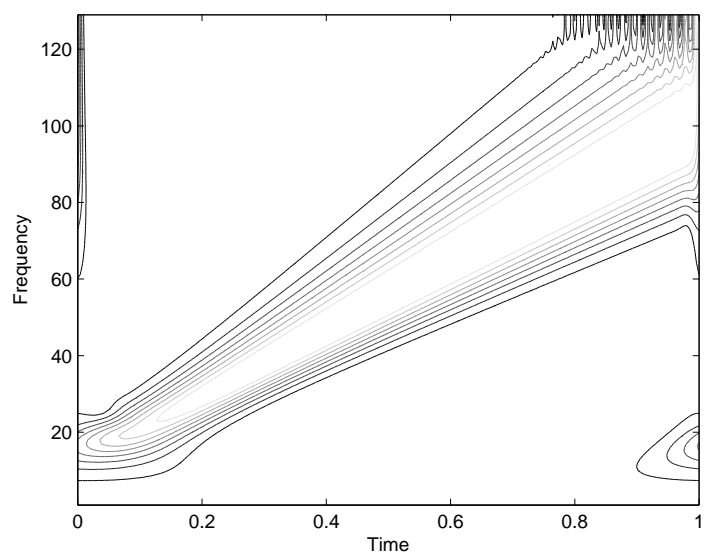

Figure 3. Time Frequency representation of a linear chirp signal using original $\mathrm{S}$ transform

remains stationary. Thus the S-transform retains the absolute phase of the signal [7].

\subsection{Generalized $S$ Transform}

Fig. 3 shows the TFR of a linear chirp signal using (4). There is a spread of energy with increasing frequency. McFadden et al. [4] and later Pinnegar and Mansinha [7] introduced a generalized S-transform which has a greater control over the window function. The generalized S transform is given by

$$
S(\tau, f, \beta)=\int_{-\infty}^{\infty} x(t) w(\tau-t, f, \beta) e^{-j 2 \pi f t} d t
$$

where $w$ is the window function of the $\mathbf{S}$ transform and $\beta$ denotes the set of parameters that determine the shape and property of the window function. For the Gaussian window $w_{G S}, \gamma$ is the only parameter in $\beta$, and from (2) and (3), $\gamma=\sigma$

$$
w(\tau-t, f, \gamma)=\frac{|f|}{\gamma \sqrt{2} \pi} \exp \left[-\frac{f^{2}(\tau-t)^{2}}{2 \gamma^{2}}\right]
$$

\subsection{Modified S Transform}

In this paper, we choose a scaling function for the gaussian window which determines $\gamma$ in (9). Thus

$$
\gamma(f)=\eta f+b
$$

where $\eta$ is the slope and $b$ is the intercept. The resolution in time and in frequency depends on both $\eta$ and $b$. We have determined usable values of $\eta$ and $b$ by trial and error. The modified $\mathrm{S}$ transform becomes

$$
S(\tau, f, \eta, b)=\int_{-\infty}^{\infty} x(t) \frac{|f|}{\sqrt{2} \pi(\eta f+b)} e^{-\frac{(\tau-t)^{2} f^{2}}{2(\eta f+b)^{2}}} e^{-j 2 \pi f t} d t
$$




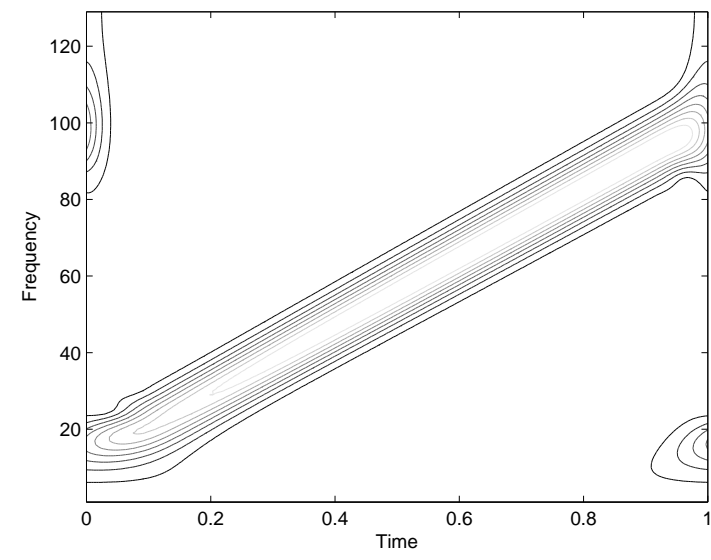

Figure 4. Time frequency representation of a linear chirp signal using Modified $S$ transform, which has a better energy concentration in the TF plane compared to original $\mathrm{S}$ transform

The modified $\mathrm{S}$ transform also satisfies the normalization condition for $\mathrm{S}$ transform windows and hence is invertible. The parameter $\gamma$ represents the number of periods of the Fourier sinusoid that can be contained within one standard deviation of the Gaussian window. The time resolution, i.e. the event onset and offset times and frequency smearing, is controlled by the factor $\gamma$. If $\gamma$ is too small the Gaussian window retains very few cycles of the sinusoid. Hence the frequency resolution degrades at low frequencies. If $\gamma$ is too high the window retains more sinusoids within it and as a result the time resolution degrades at high frequencies. It indicates that the $\gamma$ value should be varied with care for a better energy distribution in the time-frequency plane.

A typical range of $\eta$ is $0.25-0.5$ and $b$ is $0.5-3$.The variation of width of window with $\gamma$ for a particular frequency component $(25 \mathrm{~Hz})$ is illustrated in Fig. 5.

\section{The Filtering Approach}

Fig. 6 shows the TFR of the time series $h(t)$, using the modified $\mathrm{S}$ transform with the parameters $\eta=0.25$ and $b=1.9$. On the TFR there appears a low magnitude background $t$ - $f$ spectrum, with visible high magnitude spectral $t$ - $f$ components, which are the two chirp waveforms.

The filtering procedure is performed in two stages. In the first phase, the background noise is removed and then in the second stage we remove the time limited and band limited noise components of the time series.

\subsection{Background Noise Removal}

A low order 'best fit' surface is first developed by least square fitting of the $|S(\tau, f)|$. The quadratic best fit surface

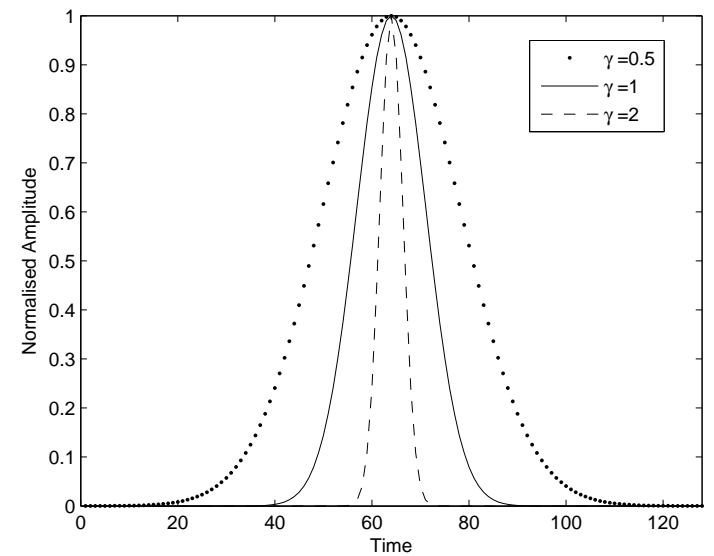

Figure 5. Variation of window width with $\gamma$ for a particular frequency $(25 \mathrm{~Hz})$

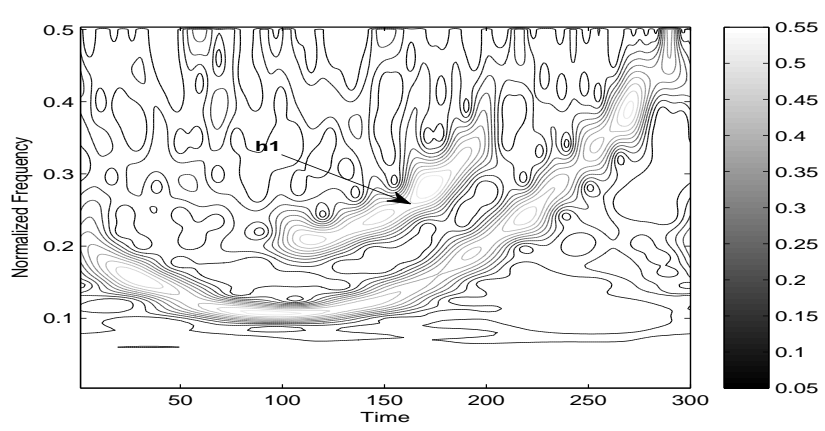

Figure 6. TFR of the time series $h(t)$ using modified S transform

obtained is shown in Fig. 7. This surface acts as a reference for removing background noise. The difference between the $\mathrm{S}$ transform plane and the best fit quadratic surface is derived to form an intermediate surface. All data on the on the intermediate surface, which are less than three standard deviations are considered as part of the background noise spectrum and are removed from the $\mathrm{S}$ transform plane. Fig. 8 depicts the TFR of $h(t)$ after removal of the background noise utilizing this criterion.

\subsection{Localised Noise Filtering}

The $\mathrm{S}$ transform plane obtained after background noise removal can be used as a foundation for filtering out localized unwanted signal elements. The ST plane is converted to an image, and the edges of the image are determined using a 'Laplacian for Gaussian' edge detection technique. The Laplacian is a 2-D isotropic measure of the $2^{\text {nd }}$ spatial derivative of an image. The Laplacian of an image highlights regions of rapid intensity change. The Laplacian is applied to the image after smoothing the image with a spatial Gaussian 


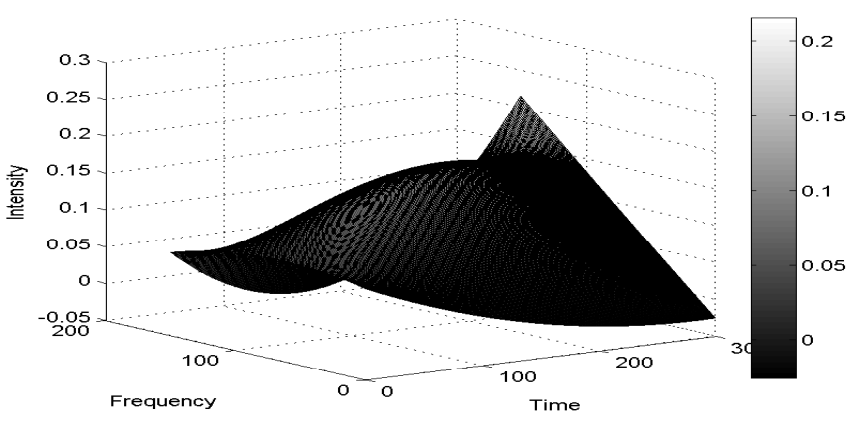

Figure 7. A quadratic best fit surface to the $S$ transform of $h(t)$, which is used as a reference for filtering out background noise

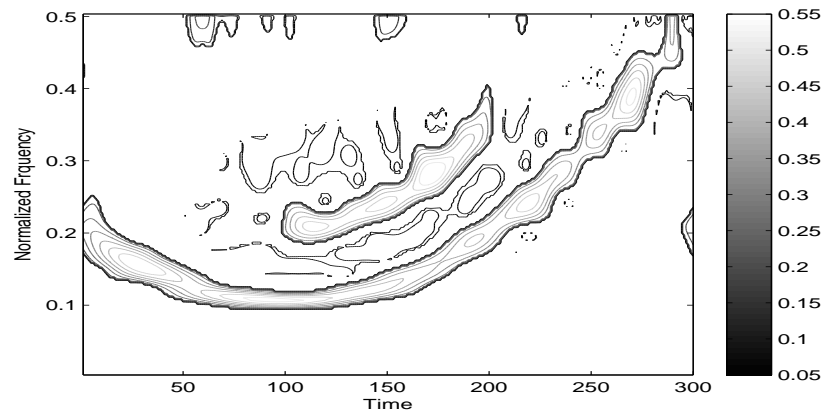

Figure 8. Time frequency distribution of $h(t)$ after removing background noise

filter in order to reduce its sensitivity to noise. If $I(x, y)$ denote the pixel intensity values of an image, the Laplacian, $L(x, y)$ can be calculated as:

$$
L(x, y)=\frac{\partial^{2} I}{\partial x^{2}}+\frac{\partial^{2} I}{\partial y^{2}}
$$

This operation produces an image that shows the edges of the signal and noise area as closed loops. The signal signatures which needed to be retained are 'whiteouted' using image filling techniques. Morphological dilation [9] is applied on the image to slightly 'grow' the signal regions in all directions on the image. The growth is intentionally made more prominent in the frequency direction of the $\mathrm{S}$ transform plane to reduce the artifacts of boxcar type filtering. The image after dilation forms a filter mask as shown in Fig. 9. The product of the $\mathrm{S}$ transform surface and the filter mask removes the noise signatures. An Inverse $S$ transform of the newly obtained $\mathrm{S}$ transform plane using (7) gives the filtered signal.

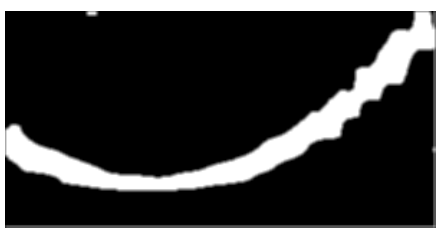

Figure 9. The time frequency filter mask developed to filter out time limited and band limited noise of $h(t)$

\section{Discussion and Conclusion}

The results of this two stage filtration are checked with the $\mathrm{S}$ transform of the filtered signal (Fig.10). From the TFR, it is clear that the background noise as well as the time limited and band limited noise that were present in $h(t)$ have been successfully removed and no extra signal components have been introduced.

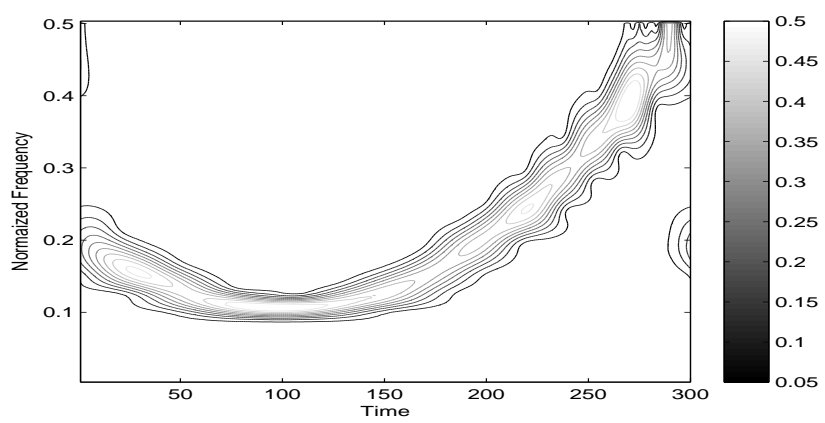

Figure 10. $\mathbf{S}$ transform of $h(t)$ after removing both the background noise and the localized noise

A second check is through the Mean Square Error (MSE) between the difference of the original and the filtered time series. The MSE is defined as

$$
M S E=\frac{\sum_{N}[h(t)-\widetilde{h}(t)]^{2}}{N}
$$

where $h(t)$ is the original time series, $\widetilde{h}(t)$ is the filtered time series and $N$ is the length of the time series. Table 1 shows the MSE comparison for different Signal to Noise Ratios(SNRs).

This paper introduced a Modified S transform which has a better energy concentration in the time frequency plane compared to the original S transform. We have successfully demonstrated a new filtering scheme to remove background noise and localized noise from noisy time series. More work needs to be done to make this method operate for highly noisy signals. 


\begin{tabular}{|c|c|}
\hline SNR (dB) & MSE \\
\hline-6 & 0.0456 \\
-3 & 0.0261 \\
3 & 0.0128 \\
10 & 0.0102 \\
20 & 0.0100 \\
30 & 0.0100 \\
40 & 0.0100 \\
\hline
\end{tabular}

Table 1. Error Analysis

\section{Acknowledgment}

The authors would like to place on record their sincere gratitude to the Department of Foreign Affairs and International Trade (DFAIT), Canada, the University of Western Ontario, Canada and the National Institute of Technology, Rourkela, India for facilitating this project. The work of KFT was supported by an NSERC Discovery Grant.

\section{References}

[1] C. R. Pinnegar, Time-frequency and time-time filtering with the S-transform and TT-transform, Digital Signal Processing 15, pp:604-620, 2005.

[2] M. Schimmel and J. Gallart, The Inverse S-Transform in filters with Time-Frequency Localization,IEEE Trans. Signal Processing 55 (11), pp:4417-4422, 2005.
[3] Stockwell RG, Mansinha L, Lowe RP Localisation of the complex spectrum: the $S$ transform, IEEE Trans Signal Processing, Vol 44(4),pp:998-1001,1996.

[4] P. D.McFadden, J. G. Cook, and L. M. Forster, Decomposition of gear vibration signals by the generalized $S$ transform,Mechanical Systems and Signal Processing, vol. 13, no. 5, pp:691-707, 1999.

[5] P.K. Dash, S.R. Samantaray, G. Panda and B.K. Panigrahi, Power transformer protection using $S$-transform with complex window and pattern recognition approach, IET Gener. Transm. Distrib., Vol. 1, pp:278-286, 2007.

[6] Chien-Chun Huang, Sheng-Fu Liang, Ming-Shing Young and Fu-Zen Shaw,A novel application of the S-transform in removing powerline interference from biomedical signals,Physiological Measurement,30,pp: 13-27,2009.

[7] C. Robert Pinnegar and Lalu Mansinha,The S-transform with windows of arbitrary and varying shape, Geophysics, Vol. 68, No. 1 ,pp:381-385,2003.

[8] Mansinha, L., Stockwell, R. G., and Lowe, R. P., Pattern analysis with two-dimensional spectral localisation: Applications of two-dimensional S-transforms, Physica A, 239,pp:286295,1997.

[9] R. M. Haralick ,S. R. Sternberg and X. Zhuang, Image analysis using mathematical morphology, IEEE Transactions on Pattern Analysis and Machine Intelligence,9,pp: 532-550, 1987. 